\title{
Epipolar Geometry for the Rectification of Cubic Panoramas
}

\author{
Florian Kangni and Robert Laganière \\ VIVA Research lab \\ School of Information Technology and Engineering \\ University of Ottawa \\ Ottawa, Ontario, CANADA K1N 6N5 \\ fkangni,laganier@site.uottawa.ca
}

\begin{abstract}
Image panoramas are of importance for virtual navigation in remote or synthetic environments. To process these panoramas, different representations have been proposed; this paper presents a study of cubic panoramas. Standard projective geometry concepts are adapted to cubic panoramas to derive the notions of fundamental matrix, essential matrix and the equivalent of stereo rectification. Methods and results are presented which could be very helpful in obtaining solutions to disparity estimation, pose estimation and view interpolation problems in the context of cubic panoramas.
\end{abstract}

\section{Introduction}

The increase of interest for image panoramas resides in the attractive and immersive possibilities they offer. In remote visualization applications, panoramas allow observing a scene from various viewing directions. When a series of panoramas, taken at different locations, are available, then very realistic exploration experiences can be achieved by virtually navigating from one panorama to another.

For the processing and storage of panoramic images, several representations have been proposed. This work focuses on a cubic representation of the image panoramas. The idea has been proposed by Fiala in [3] and is also used by Apple in their QuickTime panorama viewer [10]. $\mathrm{Cu}-$ bic panoramas offer numerous advantages that make them attractive for our study: storage and rendering are facilitated, they can be equivalently handled as set of perspective images, it exists intrinsic relationships between the faces, implicit calibration information is available, etc.

Cubes can therefore simplify one's approach to multiview image analysis; for instance, approaches inspired by classical stereo algorithms still apply. We study, in this paper, the projective relationships between cubes and extract the usual entities involved in two-view imagery: the fundamental matrices and the essential matrix.

The fundamental matrices study considers each face of the cubes as a distinct perspective image. Our analysis identifies the intrinsic relationships that link these images and is a first step in the expression of the epipolar geometry applied to cubic panoramas. More interestingly, the essential matrix reveals to be a much more convenient and compact representation of the multi-cube geometry. The essential matrix applied to cubic panoramas has been discussed by Fiala and Roth in [4]. They also proposed a method for adjacent panorama alignment in which they assume that the translational component can be neglected. The rectification method presented here is a generalized solution of the alignment problem that applies to any cube configuration. Cubic panoramas rectification is also of interest to disparity estimation, view interpolation and pose estimation problems. It is carried out here on the model of stereo rectification discussed among others in [8] and [6].

This work is part of the NAVIRE research project ${ }^{1}$ at the University of Ottawa. This research aims at developing the necessary technology to allow a user to virtually walk through in an image-based representation of a remote environment. The next section briefly discusses cubic panorama acquisition. Section 3 describes the fundamental matrix relations. Section 4 concerns the estimation of the essential matrix in the context of cubic panoramas. Section 5 describes the rectification method. Section 6 is a conclusion.

\section{Cubic panoramas acquisition}

Cubic panoramas being the subject of the study it is necessary to provide some elements on the way they are obtained. The capture of the images that are composed into panoramas is done using the Ladybug camera from Point

\footnotetext{
${ }^{1}$ visit the website www.site.uottawa.ca/research/viva/projects/ibr/ for more information on this project
} 


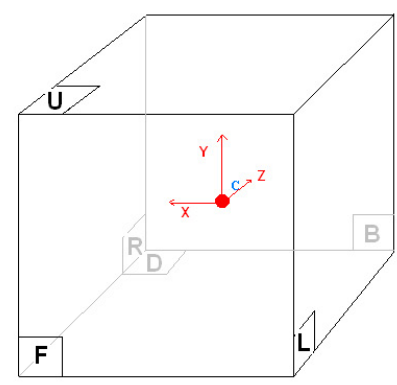

Figure 1. Cube reference frame.

Grey Research. It is essentially a camera composed of 6 sensors $(1024 \times 768$ pixels each), 5 laterals and 1 pointing upwards that capture a view of the world at 360 degrees around the azimuth completed by a top view. Since the camera's sensors have been accurately calibrated, it is possible to fuse the six images to form an almost complete spherical panorama. This panorama can therefore be considered to have been produced by a central projection camera that collects all light ray coming from all directions, incident on a point in space. The resulting two-dimensional plenoptic function can then be re-projected on any type of surface. We use here a cubic representation that have been shown to be easily manipulable and that can be rendered very efficiently on standard graphic hardware [1]. The fact that a cubic panorama is effectively made of six identical faces, each of them acting as a standard perspective projection camera with $90^{\circ}$ field of view, makes the representation very convenient to handle; all standard linear projective geometry concepts still being applicable.

One such cube is shown in Figure 2, laid out in a cross pattern with the faces in the order (from top to bottom and left to right): up, left, front, right, back, down. The panoramas we used have been generated from the real images of the Ladybug sensor using a procedure that is explained in [1]. The reference frame chosen in our study is the standard openGL frame, located at the projection center, inside the cube with the $x$ axis pointing toward the "right" face, the $y$ axis toward the "up" face and the $\mathrm{z}$ axis consequently pointing toward the "back" face (cf. Figure 1).

\section{Fundamental Matrices in Cubic Panora- mas}

This section introduces the concept of epipolar geometry applied to cubic panoramas through the study of the fundamental matrices. Essentially the 3D structure of the cube is used to derive interesting properties between faces of a same cube and between corresponding faces of two cubes. In particular, the intrinsic relationship between the faces ap-

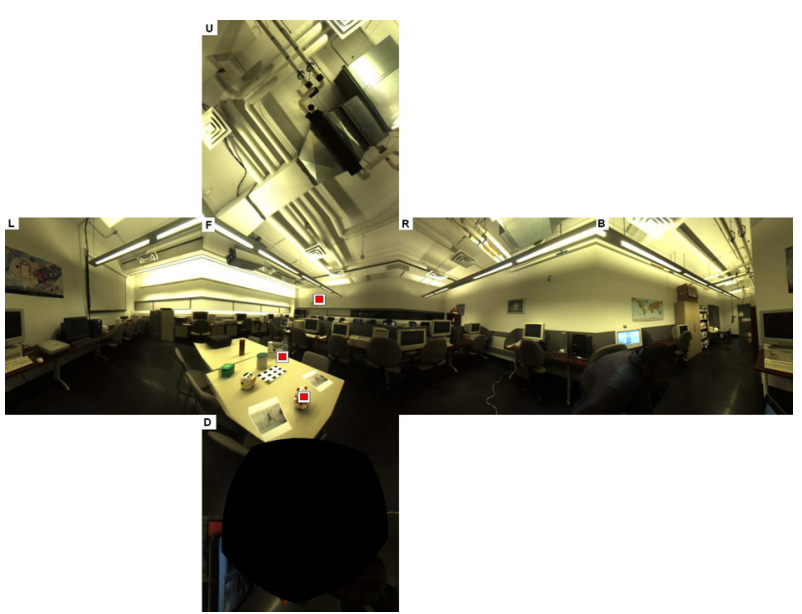

Figure 2. Example of a cube laid out in a cross pattern

pears to be very useful in our derivations as will be seen in the following sections.

\subsection{Notations}

Let us consider two cubes $(C)$ and $\left(C^{\prime}\right)$ and let us designate each face of the cube by a label $i \in\{\mathrm{U}, \mathrm{L}, \mathrm{F}, \mathrm{R}, \mathrm{B}, \mathrm{D}\}$ with $\mathrm{U}$ standing for the up face, $\mathrm{L}$ for the left face and so on.

The projection of 3D point $\mathbf{X}=(X, Y, Z)^{T}$ on a face $i$ of the cube is noted $\tilde{\mathbf{x}}_{i}=\left(x_{i}, y_{i}, 1\right)^{T} . R_{x}(\theta)$ stands for a rotation around the axis $x$ of amplitude $\theta$ and $t$ stands for a translation vector. We denote $P_{i}$ the projection matrix for a given face and $K$ the common calibration matrix since the 6 faces have identical characteristics. We can therefore write: $P_{i}=K\left[R_{i} \mid t_{i}\right]$.

\subsection{Homography between two faces}

Let us consider in this section the face $\mathrm{F}$ and the face $i$ for any $i$ in $\{\mathrm{U}, . ., \mathrm{B}\}$. Without loss of generality, if we consider the world coordinate system to be attached to the center of the cube with axis "aligned" with the front face $\mathrm{F}$, the respective projection matrices for $\mathrm{F}$ and $i$ are the following:

$$
P_{\mathrm{F}}=K\left[I_{3} \mid 0\right]
$$

and

$$
P_{i}=K\left[R_{i} \mid 0\right]
$$

with $I_{3}$ the identity matrix of order 3 . The projection matrices $P_{\mathrm{F}}$ and $P_{i}$ differ only by a rotation $R_{i}$ since the focal center is the same for all faces. Note that for all $i$, we have $R_{i}=R_{\text {axis }}(\theta)$ with $\theta$ in the set $\left\{\frac{-\pi}{2}, 0, \frac{\pi}{2}, \pi\right\}$ and axis standing for $x, y$, or $z$ depending on the face : for example 
$R_{\mathrm{R}}=R_{x}\left(\frac{-\pi}{2}\right)$. All these matrices are detailed in appendix A. For a point $\mathbf{X}$ in space, its projections $\tilde{\mathrm{X}}_{\mathrm{F}}$ and $\tilde{\mathrm{x}}_{i}$, are given by :

$$
\tilde{\mathrm{X}}_{\mathrm{F}}=P_{\mathrm{F}} \mathbf{X}
$$

and

$$
\tilde{\mathbf{x}}_{i}=P_{i} \mathbf{X}
$$

From (1) and (3) we can extract an expression for $\mathbf{X}$ as done in [7]:

$$
\mathbf{X}=\left(\begin{array}{c}
Z K^{-1} \tilde{\mathbf{x}}_{\mathrm{F}} \\
1
\end{array}\right)
$$

By replacing $\mathbf{X}$ in (4) by its expression in (5), we obtain :

$$
\tilde{\mathrm{x}}_{i}=Z K R_{i} K^{-1} \tilde{\mathrm{x}}_{\mathrm{F}}
$$

Let us note :

$$
H_{i}=K R_{i} K^{-1}
$$

As a consequence, (6) becomes :

$$
\tilde{\mathrm{x}}_{i}=Z H_{i} \tilde{\mathrm{x}}_{\mathrm{F}}
$$

which, in projective space, is equivalent to $(Z$ being a scalar):

$$
\tilde{\mathrm{X}}_{i}=H_{i} \tilde{\mathrm{x}}_{\mathrm{F}}
$$

In the general case, i.e between any two faces $i$ and $j$, the previous equation is rewritten as:

$$
\tilde{\mathrm{x}}_{j}=K R_{j} R_{i}^{-1} K^{-1} \tilde{\mathrm{x}}_{i}=H_{i j} \tilde{\mathrm{x}}_{i}
$$

with

$$
H_{i j}=K R_{j} R_{i}^{-1} K^{-1}
$$

\subsection{Fundamental Matrices between two Cubes}

The standard 8-point algorithm mentioned in $[6,12]$ can be used to compute the fundamental matrix between a pair of faces given a set of point correspondences. The rest of the procedure explained below is carried on once one of the possible six fundamental matrices is computed.

For two corresponding faces $i$ in two cubes, we have:

$$
\tilde{\mathrm{x}}_{i}^{\prime T} F_{i} \tilde{\mathrm{x}}_{i}=0
$$

where $\tilde{\mathrm{x}}_{i}$ and $\tilde{\mathrm{x}}_{i}^{\prime}$ are matches visible on both face $i$ of $(C)$ and face $i$ of $\left(C^{\prime}\right)$ respectively (matches visible in different faces are not considered here since each face is considered as a distinct camera; this case will be handled in the computation of the essential matrix presented in Section 4). Inserting (9) in (12) yields:

$$
\tilde{\mathrm{x}}_{\mathrm{F}}^{\prime T} H_{i}^{T} F_{i} H_{i} \tilde{\mathrm{X}}_{\mathrm{F}}=0
$$

From which we conclude :

$$
F_{\mathrm{F}}=H_{i}^{T} F_{i} H_{i}
$$

or

$$
F_{i}=H_{i}^{-T} F_{\mathrm{F}} H_{i}^{-1}
$$

with $H_{i}=K R_{i} K^{-1}$ as defined in (7). A similar equation that links the $F$ matrices of any two faces $i$ and $j$ can be obtained; we thus have:

$$
F_{j}=H_{i j}^{-T} F_{i} H_{i j}^{-1}
$$

with $H_{i j}$ defined in (11).

\subsection{Observations}

We have seen so far that an interesting intrinsic relationship existed between the fundamental matrices of the faces of two cubes. An important point to note is the necessity of knowing the calibration matrix $K$ associated with each face. As mentioned in sections 1 and 2, an advantage of the cubic representation resides in the constraints it introduces. The calibration matrix $K$ can indeed be deduced from $L$, the size of a cube in pixels. The image plane is at a distance $\frac{L}{2}$, the principal point of each image plane is always at $\left(\frac{L}{2}, \frac{L}{2}\right)$. Thus we can write :

$$
K=\left(\begin{array}{ccc}
\frac{L}{2} & 0 & \frac{L}{2} \\
0 & -\frac{L}{2} & \frac{L}{2} \\
0 & 0 & 1
\end{array}\right)
$$

Another aspect of the epipolar geometry which provides a verification mean for the previous computation of the fundamental matrices is the epipolar plane. Since all cube faces share the same projection center, this epipolar plane is the same for all faces. The epipolar lines on each face are therefore obtained by intersecting the cube with the epipolar plane generated by a given image point in the other cube. As a consequence, the epipolar lines on the faces of a cube should be connected across adjacent faces.

Finally, to obtain the epipolar lines around the cube, it is important to note that:

a. for a point $\tilde{\mathrm{x}}_{i}$ in face $i$ of $(C)$, its corresponding line in face $i$ of $\left(C^{\prime}\right)$ is $l_{i i}=F_{i} \tilde{\mathrm{x}}_{i}$. This is the classical case met in state of the art stereo.

b. for a point $\tilde{\mathrm{x}}_{i}$ in face $i$, its corresponding line in face $j$ of $\left(C^{\prime}\right)$ is given by $l_{i j}=F_{j} \tilde{\mathbf{x}}_{j}=F_{j} H_{i j} \tilde{\mathbf{x}}_{i}$ with $j \neq i$ and $H_{i j}$ given by expression (11). This corresponds to re-projecting the point to the face of interest before applying the appropriate fundamental matrix.

Figure 3 shows a set of epipolar lines relating the six fundamental matrices of a cube $(C)$, not shown here, with 


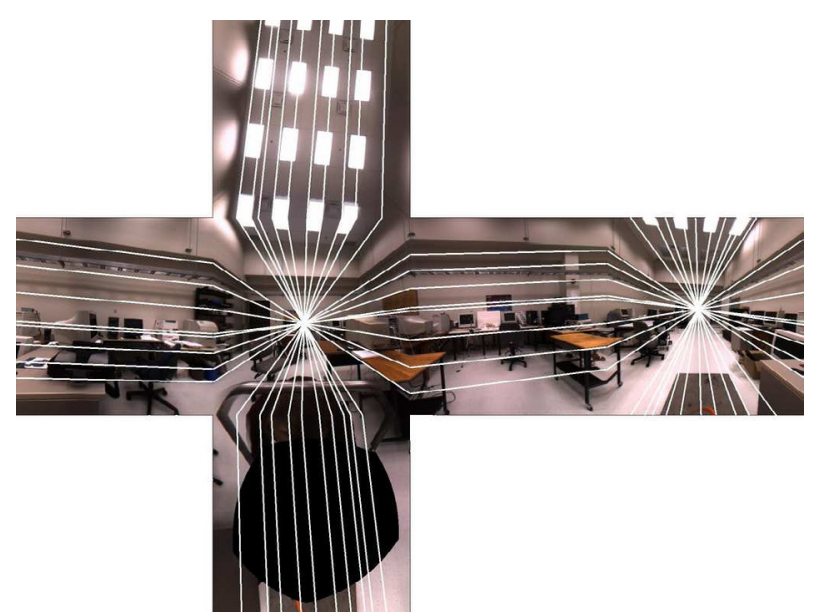

Figure 3. Epipolar lines over a cube.

a cube $\left(C^{\prime}\right)$ shown on this figure. The fact that there is a double epipole visible on the frontal and backward faces indicates a configuration where the two cubes are aligned one in front of the other. The result is also visually satisfying guaranteeing we are indeed witnessing intersections of a cube by a plane with the noticeable and expected connected line pattern.

\section{The Essential Matrix}

In the previous sections we looked at the cubes' epipolar geometry based on a stereo approach where each face of a cube was considered as a distinct camera. The notion of essential matrix constitutes a more compact form of all relationships between the cubes faces. The essential matrix concept applied to cube has also been discussed by Fiala and Roth in [4].

a. By definition [7, 12], the essential matrix embeds more information than its counterpart discussed in the previous section. The calibration needs to be known to be able to estimate E. Nonetheless, in the case of the cube, we have seen that the calibration matrix is implicitly known. Estimating $\mathrm{E}$ then becomes a matter of solving the classical problem $m^{\prime T} E m=0$ with for example the 8-point algorithm used previously as recommended in [7].

b. An advantage of the cube representation resides in the fact that the coordinates of a point on a face of the cube can be expressed by the corresponding 3D coordinates of that point with respect to the reference frame displayed on Figure 1. Each image point on a cube face is therefore mapped to a 3D point coordinate $p$ that is function of the point position on the face and of the cube side it is on. c. Approaching the problem from the point of view of $\mathrm{E}$ has the advantage of making us work directly with the epipolar plane. Indeed for any point $p$ of $C, E p$ is a plane in $C^{\prime}$ - the epipolar plane - intersecting the cubic panorama (the complete derivation is given in [4]): E allows a closed form recovery of the epipolar geometry.

\subsection{E Estimation}

In the estimation of $E$, the coordinates of each matched point must first be converted from its projective coordinates to the cube coordinate system by applying the appropriate transformation, $p=T_{i} \tilde{\mathrm{x}}$. All $T_{i}$ are given in Appendix B. The 8-point algorithm is then applied to solve for $\mathrm{E}$ in the well-known classical equation $p^{\prime T} E p=0$ for a given set of matches. Nonetheless two important remarks are to be mentioned here.

First, the estimation algorithm typically requires a normalization procedure [5] that is simplified in our case by the fact that the points all belong to a cube. Indeed, assuming that all the feature points are uniformly distributed over the faces, the centroid of the image points expressed in cube coordinates should be close the origin of the cube reference frame. The normalization step will therefore just be a scaling of the 3 coordinates of all $p_{k}$ by the maximum possible coordinate value which is $\frac{L}{2}$.

Second, the singular values $E$ should be equal: if $a$ and $b$ are the two singular values of the best estimate of $E$, we consequently have to force both of them to be equal to $s=$ $\frac{a+b}{2}$; the third singular value being null. For more details the reader is advised to consult [2].

\section{$4.2 \quad$ Results}

This section shows simply the consistency between the approach based on fundamental matrices and the one relying on E. The epipolar lines associated with any point $\tilde{x}$ on a face of the cube can be obtained by intersecting the epipolar plane $E p$ with the corresponding cube. As explained in Section 3, they can also be obtained from the knowledge of one of the cube face's fundamental matrix. To validate the consistency of the two approaches, few epipolar lines are displayed on the cube image shown in figure 5 that has been matched with the cube of Figure 2. These lines correspond to the three image points identified by square markers on the frontal face of the image in Figure 2. 


\section{Cubic Panorama Rectification}

\subsection{Principle}

To rectify the images of a stereo pair, the procedure generally consists in sending the epipoles to infinity in the $x$ direction. This can be achieved by applying two rectifying homographies $h$ and $h^{\prime}$ to each image of the stereo pair $[6,8]$. The net result is a set of (horizontal) parallel epipolar lines. In the case of cubic panoramas, the rectification process would consist in making the corresponding cube faces parallel to each other. To achieve this goal, a principle ana$\log$ to [8] is followed that, here, consists in finding the two rotations $R_{1}$ and $R_{1}^{\prime}$ that align the cubes in the preferred configuration.

The resulting configuration can be seen on Figure 4. It basically shows that the rotations $R_{1}$ and $R_{1}^{\prime}$ are such that the $x$ axis of both cubes coordinate systems merge into a common axis between both cubes going through both centers (baseline axis): the difference between the cubes $(C)$ and $\left(C^{\prime}\right)$ becomes only translational. In such a configuration $[7,8]$ showed that the corresponding essential matrix is

$$
E_{\text {rect }}=\left[(1,0,0)^{T}\right]_{x}=\left(\begin{array}{ccc}
0 & 0 & 0 \\
0 & 0 & -1 \\
0 & 1 & 0
\end{array}\right)
$$

Thus, we want to find $R_{1}$ and $R_{1}^{\prime}$ such that, if $E$ is the essential matrix between $(C)$ and $\left(C^{\prime}\right)$, then:

$$
R_{1}^{\prime-T} E_{\text {rect }} R_{1}^{-1}=E
$$

or

$$
E_{\text {rect }}=R_{1}^{\prime T} E R_{1}
$$

Compared to the stereo case, we have homographies becoming equivalent rotations and the fundamental matrices replaced by their essential counterparts.

The interest of such a rectification procedure resides in the simplifications it introduces. For example such alignment could greatly facilitate cubic panorama interpolation. It could also help stabilize a linear sequence of cubes (as done in [4]) by aligning them along a common direction, thus reducing navigation jitters. There is also a great benefit in applying dense stereo algorithms to rectified images. Finally, the fact that cube rectification eliminates the rotational component between cubes could greatly simplify the pose estimation process as the only unknowns become the relative translations between a set of cubes.

The next subsections detail the proposed cube rectification procedure.

\subsection{Rotation $R_{1}$}

To recover this rotation, we only need to align the $x$-axis of the reference frame of $(C)$ with the baseline axis of the

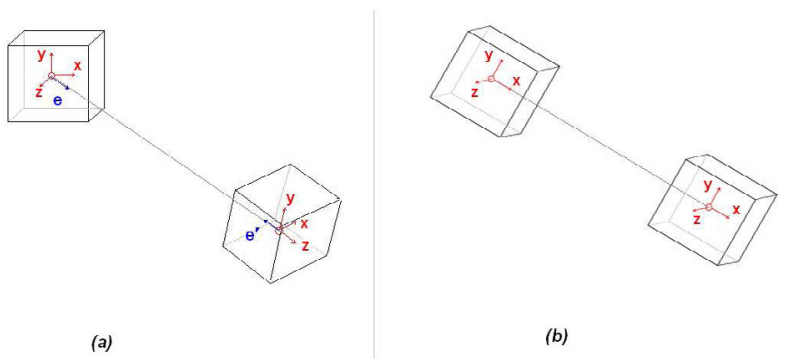

Figure 4. (a) Cubes in general configuration (b) Aligned cubes

cube pair. This is done by first recovering the epipole vector $e$ which gives the direction $C \rightarrow C^{\prime}$ in $(C)$ reference frame. We know that $E e=0$ meaning that $e$ can be obtained from the SVD decomposition of $E$. The next step is to geometrically align the $x$-axis $(1,0,0)^{T}$ with $e=\left(e_{x}, e_{y}, e_{z}\right)$. Two rotations $R_{z}(\theta)$ and $R_{y}(\phi)$ suffice and are composed to find $R_{1}$ such that:

$$
R_{1}=R_{y}(\phi) R_{z}(\theta)
$$

with the constraint:

$$
R_{1}(1,0,0)^{T}=e
$$

which allows us to recover :

$$
\theta=\arcsin \left(e_{y}\right)
$$

and

$$
\phi=\arctan \left(\frac{e_{z}}{e_{x}}\right)
$$

\section{$5.3 \quad$ Rotation $R_{1}^{\prime}$}

The exact same procedure as above is applied to recover $R_{1}^{\prime}$, the only difference being the $x$-axis of $\left(C^{\prime}\right)$ is aligned with $-e^{\prime}$ instead of $e^{\prime}$.

\subsection{Additional rotation $R_{2}^{\prime}$}

One could think that $R_{1}$ and $R_{1}^{\prime}$ provide the final solution to the problem. As a matter of fact they only allow the $(C)$ and $\left(C^{\prime}\right) x$-axes to be aligned. Nothing then guarantees the complete face alignment. This explains the need for an additional rotation $R_{2}^{\prime}$ that will supplement $R_{1}^{\prime}$ to force the face alignment of $\left(C^{\prime}\right)$ with respect to $(C)$. This problem is formulated as follows :

$$
\left(R_{2}^{\prime} R_{1}^{\prime}\right)^{-T} E_{\text {rect }} R_{1}^{-1}=\alpha E
$$

We know that the additional rotation will be around the axis $-e^{\prime}$. We also know $E_{\text {rect }}, R_{1}, R_{1}^{\prime}$ and $E$. The fact that we 
know the axis of rotation reduces the number of unknowns in (24) to 2: the rotation angle $\theta$ around the axis $-e^{\prime}$ and the scaling factor $\alpha$. The equation can then be considered of the form :

$$
f(\theta, \alpha)=0
$$

It is a minimization problem that we solved using the simplex algorithm [9].

The rotation $R_{1}^{\prime}$ in section 5.3 is multiplied by $R_{2}^{\prime}$ to obtain the final rotation to apply to $\left(C^{\prime}\right)$.

\subsection{Rectification Example}

Tests were conducted on two cubes in arbitrary configuration. Figure 6 displays a 3D view of the situation before and after alignment generate. To create the original configuration on the left, the rotation between the cubes is extracted from the essential matrix following [2]. The rectifying rotations were then applied to the two cubes. The resulting rectified configuration shown is what was expected in terms of $x$-axis and faces alignment.

One should note that one critical step is the approximation of $R_{2}^{\prime}$ through the minimization of 25 which will practically give only an approximate compensating angle. Depending on the accuracy of the minimization technique chosen and in some cases on the initial estimates of the angle, the result may vary.

\section{Conclusion}

This paper presented a study of important aspects of the epipolar geometry in the case of a particular format of images panoramas.

Cubic panoramas have proved by many of their advantages to be worthy of interest. Among these advantages intrinsic relationships between faces and implicit calibration were used to derive important epipolar concepts through fundamental matrices linking pairs of matching faces and the essential matrix. From these results, one can then use the epipolar constraint deriving from these matrices, to define a reliable matching procedure following a RANSAC scheme such as the one proposed in [11]

Finally, from the essential matrix we derive the equivalent principle of stereo rectification in the case of cubes and we showed how rectification can eliminate the rotational component in a multi-cube configuration.

\section{Appendix A : rotations matrices}

The rotations $R_{i}$ mentioned in the article are obtained by simply observing the frame on figure 1 . To align the frame to each face $i$, a trivial rotation needs to be applied. We thus can derive the following expressions for each face :

$$
\begin{gathered}
R_{\mathrm{U}}=R_{x}\left(\frac{\pi}{2}\right)=\left(\begin{array}{ccc}
1 & 0 & 0 \\
0 & 0 & -1 \\
0 & 1 & 0
\end{array}\right) \\
R_{\mathrm{L}}=R_{y}\left(\frac{\pi}{2}\right)=\left(\begin{array}{ccc}
0 & 0 & 1 \\
0 & 1 & 0 \\
-1 & 0 & 0
\end{array}\right) \\
R_{\mathrm{F}}=R_{x}(0)=\left(\begin{array}{ccc}
1 & 0 & 0 \\
0 & 1 & 0 \\
0 & 0 & 1
\end{array}\right) \\
R_{\mathrm{R}}=R_{y}\left(-\frac{\pi}{2}\right)=\left(\begin{array}{ccc}
0 & 0 & -1 \\
0 & 1 & 0 \\
1 & 0 & 0
\end{array}\right) \\
R_{\mathrm{B}}=R_{y}(\pi)=\left(\begin{array}{ccc}
-1 & 0 & 0 \\
0 & 1 & 0 \\
0 & 0 & -1
\end{array}\right) \\
R_{\mathrm{D}}=R_{x}\left(-\frac{\pi}{2}\right)=\left(\begin{array}{ccc}
1 & 0 & 0 \\
0 & 0 & 1 \\
0 & -1 & 0
\end{array}\right)
\end{gathered}
$$

\section{Appendix B : 3D conversion and plane inter- sections}

In the case of a cube of side $L$, faces are located at trivial coordinates with respect to the cube reference frame : $x=$ $\pm \frac{L}{2}$ for right and left faces, $y= \pm \frac{L}{2}$ for top and down faces, $z= \pm \frac{L}{2}$ for front and back faces. This allows us to convert easily $2 \mathrm{D}$ faces coordinates into $3 \mathrm{D}$ cube reference frame coordinates. In general, if $\tilde{\mathrm{x}}=(x, y, 1)^{T}$ is the point of concern, an affine transformation is applied to $x$ and $y$ to find two of the three $3 \mathrm{D}$ coordinates along the axis $x, y$ or $z$ that form the plane of the face, the third coordinate being a constant as mentioned above.

$$
\begin{aligned}
T_{\mathrm{U}} & =\left(\begin{array}{ccc}
1 & 0 & -\frac{L}{2} \\
0 & 0 & \frac{L}{2} \\
0 & -1 & \frac{L}{2}
\end{array}\right) \\
T_{\mathrm{L}} & =\left(\begin{array}{ccc}
0 & 0 & -\frac{L}{2} \\
0 & -1 & \frac{L}{2} \\
-1 & 0 & \frac{L}{2}
\end{array}\right) \\
T_{\mathrm{F}} & =\left(\begin{array}{ccc}
1 & 0 & -\frac{L}{2} \\
0 & -1 & \frac{L}{2} \\
0 & 0 & -\frac{L}{2}
\end{array}\right) \\
T_{\mathrm{R}} & =\left(\begin{array}{ccc}
0 & 0 & \frac{L}{2} \\
0 & -1 & \frac{L}{2} \\
1 & 0 & -\frac{L}{2}
\end{array}\right)
\end{aligned}
$$




\begin{tabular}{|c|c|c|c|}
\hline face & $a_{i}$ & $b_{i}$ & $c_{i}$ \\
\hline $\mathrm{U}$ & $a$ & $-c$ & $g_{\mathrm{U}}(b)$ \\
$\mathrm{L}$ & $-c$ & $-b$ & $g_{\mathrm{L}}(-a)$ \\
$\mathrm{F}$ & $a$ & $-b$ & $g_{\mathrm{F}}(-c)$ \\
$\mathrm{R}$ & $c$ & $-b$ & $g_{\mathrm{R}}(a)$ \\
$\mathrm{B}$ & $-a$ & $-b$ & $g_{\mathrm{B}}(c)$ \\
$\mathrm{D}$ & $a$ & $c$ & $g_{\mathrm{D}}(-b)$ \\
\hline
\end{tabular}

\section{Table 1. Epipolar lines as intersections of cube and epipolar plane}

$$
\begin{gathered}
T_{\mathrm{B}}=\left(\begin{array}{ccc}
-1 & 0 & \frac{L}{2} \\
0 & -1 & \frac{L}{2} \\
0 & 0 & \frac{L}{2}
\end{array}\right) \\
T_{\mathrm{D}}=\left(\begin{array}{ccc}
1 & 0 & -\frac{L}{2} \\
0 & 0 & -\frac{L}{2} \\
0 & 1 & -\frac{L}{2}
\end{array}\right)
\end{gathered}
$$

On the other hand, the fact that the faces lie at particular coordinates allows us also to find the intersections of a plane with the cube. The epipolar plane is given by $E p$ if $p$ is the point of interest. It is a plane that goes through the center of the cube and is noted $(a, b, c)$ to designate its normal. We therefore have the results presented in table 1 where the different lines $l_{i}=\left(a_{i}, b_{i}, c_{i}\right)$ lie on the faces of the cube as the intersections of the latter with the plane $(a, b, c)$. The functions $g_{i}$ in table 1 are defined as follows :

$$
g_{i}(m)=\frac{L}{2}\left(m-\left(a_{i}+b_{i}\right)\right)
$$

\section{Acknowledgements}

The authors wish to thank Alan Brunton for his help in generating the cubic panoramas, and also Akshay Bhatia for the image capture. This work has been supported by the NSERC Strategic Project Grants program.

\section{References}

[1] D. Bradley, A. Brunton, M. Fiala, and G. Roth. Imagebased navigation in real environments using panoramas. In IEEE Int. Workshop on Haptic Audio Visual Environments and their Applications, pages 103 - 108, October 2005.

[2] O. Faugeras and Q.-T. Luong. The geometry of multiple images. Cambridge University Press, ISBN: 0262062208, first edition, 2001.

[3] M. Fiala. Immersive panoramic imagery. In Canadian Conference on Computer and Robot Vision, pages 386 - 391, 2005.
[4] M. Fiala and G. Roth. Automatic alignment and graph map building of panoramas. In IEEE Int. Workshop on Haptic Audio Visual Environments and their Applications, pages 103 - 108, October 2005.

[5] R. Hartley. In defense of the 8-point algorithm. IEEE trans. Pattern Analysis and Machine Intelligence, 19:580593, 1995.

[6] R. Hartley. Theory and practice of projective rectification. Int. journal Computer Vision, 35(2):115-127, 1999.

[7] R. I. Hartley and A. Zisserman. Multiple View Geometry in Computer Vision. Cambridge University Press, ISBN: 0521540518, second edition, 2004.

[8] J. Mallon and P. Whelan. Projective rectification from the fundamental matrix. Image and Vision Computing, 23(7):643-650, July 2005.

[9] J.-P. Moreau. Functional approximations in c/c++ : tamoeba.cpp.

[10] A. Q. VR. Introduction to Quicktime VR.

[11] A. Whitehead and G. Roth. The projective vision toolkit. In Proceedings, Modelling and Simulation., pages 204-209, 2000.

[12] Z. Zhang. Determining the epipolar geometry and its uncertainty: A review. Technical Report 2927, Sophia-Antipolis Cedex, France, 1996. 


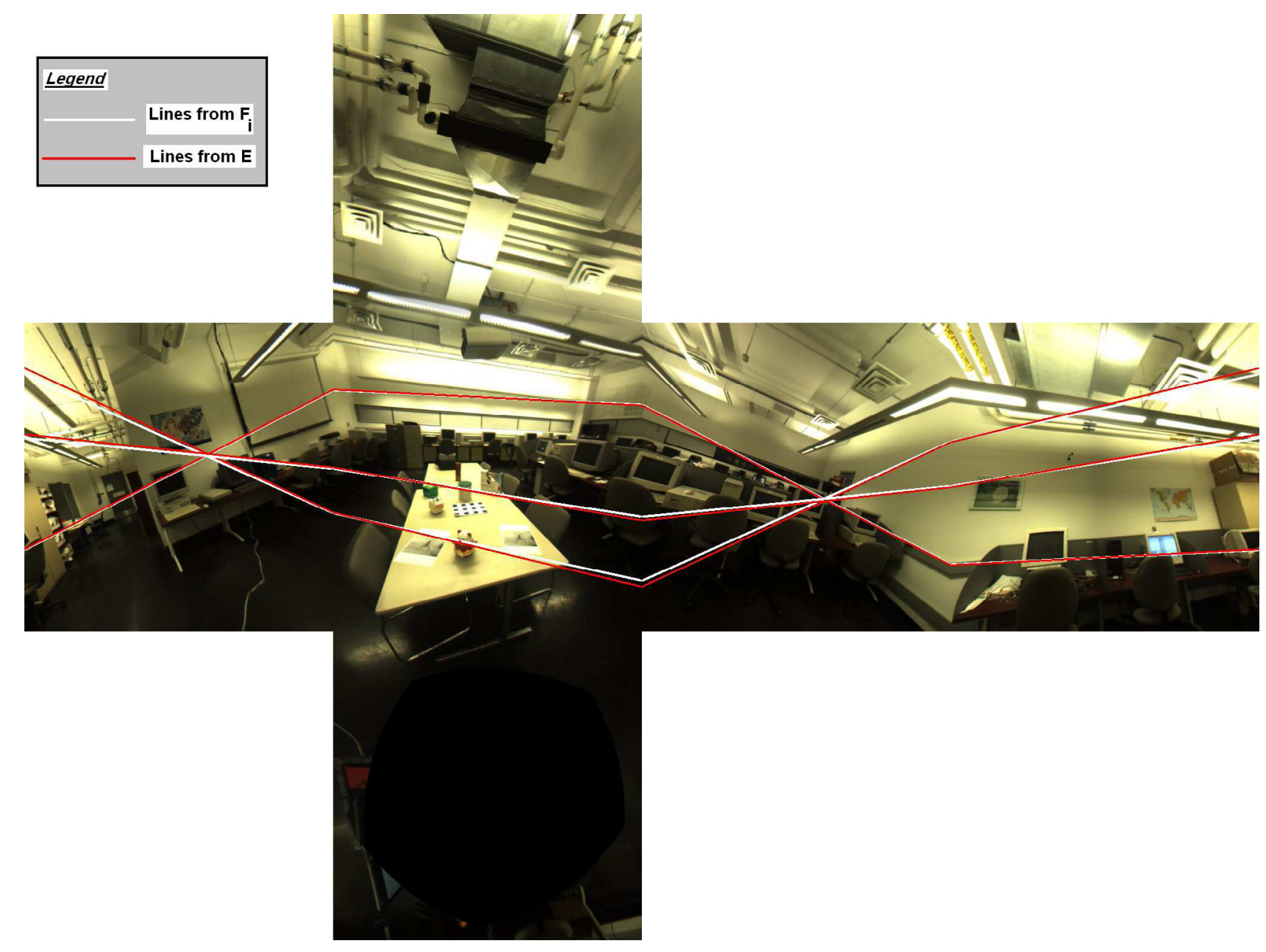

Figure 5. Epipolar lines over a cube as computed from $E$ and from the matrices $F_{i}$.
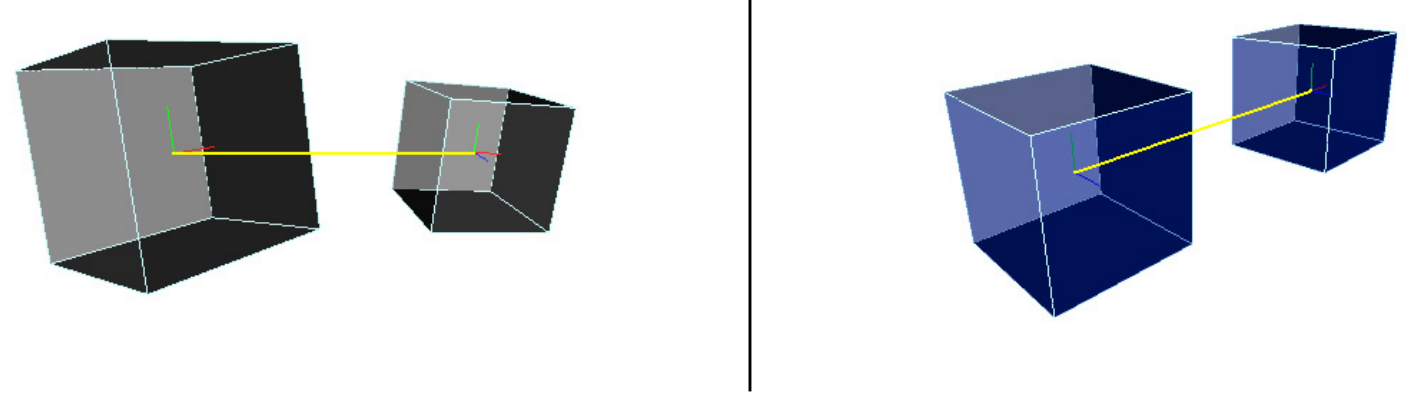

Figure 6. The estimated 3D position of the tested cube pair (figures 2 and 5). Original configuration (left). After rectification (right) 